\title{
Calculation of the hydraulic characteristics of the protective coating used in trenchless technologies for the construction and renovation of pipelines to extend their service life
}

\author{
Vladimir Orlov ${ }^{1, *}$, Sergey Zotkin $^{1}$, Irina Dezhina ${ }^{1}$ and Irina Zotkina ${ }^{1}$ \\ ${ }^{1}$ Moscow state university of civil engineering, Yaroslavskoye shosse, 26, Moscow, Russia, 129337
}

\begin{abstract}
The lifetime of the pipeline, which undergoes trenchless repair by pulling and then fixing various types of internal protective coatings inside, is determined by the strength characteristics of the two-layer structure "old pipeline + internal protective coating". Hydraulic parameters of internal protective coatings, such as surface roughness and degree of hydrophobicity (water repellency), can play a role in prolonging the life of the repaired pipeline. With a high degree of hydrophobicity of the protective coating, the contact of the transported liquid with the walls of the pipeline during its operation in the non-pressure regime decreases, which leads to a decrease in the negative impact on the walls, in particular, their attrition, interaction with the specific structure of the coating material, which in turn reduces the energy costs when transporting water. Given article deals with a mathematical description of the interaction of the surface structure (with its inherent wetting ability) and the transported liquid in a mini-stream created on an inclined open chute imitating the inner surface of a pressureless pipeline. The theoretical positions and methodology for calculating the degree of hydrophobicity are based on research conducted on the basis of frontal and coaxial (along the flow) digital photo and video. $\mathrm{T}$
\end{abstract}

\section{Introduction}

The wide application of new polymer repair materials in the form of hoses applied on the inner surface of pipes with bearing capacity has opened a new page in the practice of efficient and operative renovation of water supply and sewage pipelines [1].

The modern construction market is replete with materials that can potentially be reliable internal protective coatings for the pipeline transport of liquids and gases. Polymeric materials have not only high strength characteristics, but also allow to achieve the energy saving effect in the transportation of water due to the corresponding hydraulic characteristics of the materials used with low coefficients of hydraulic friction [2]. In

\footnotetext{
* Corresponding author: <orlov950@yandex.ru>
} 
addition, polymeric materials are not subject to gas, chemical and biocorrosion, such as steel, cast iron and reinforced concrete, which is another additional advantage over other materials used for the production of pipes [3].

In recent years, there has been some interest in the study of such materials, especially in terms of studying their hydrophobicity and its relationship to the service life of pipelines used in the field of water supply, wastewater and gas supply [4, 5]. In the construction sector, the use of unique nanomaterials is also actual, which can be used as internal coating for pipeline networks.

It should be noted that the capabilities of modern polymer and nanomaterials used as protective coatings of pipelines are unlimited thanks to their unique properties. The problem of studying the smoothest surfaces has been addressed by modern technology for a long time. Obviously, the smaller the roughness, the smoother the surface is and the less friction and wear of the surface are caused by the impact of the transported environment (natural water, sewage, etc.), as well as the higher energy efficiency of the pipelines in operation is provided. During the choosing repair materials, first of all it's necessary to rely on established criteria. Unevenness for which the ratio of the height of the protrusions $S_{c}$ to the diameter of the pipeline $D_{c}$ is $S_{c} / D_{c}<40$, is generally estimated as roughness [6]. As a rule, the height of unevennesses is measured by optical instruments (light and shadow cross-sections), by microinterference methods, using rasters (special grids with a small step and a scanning microscope).

Mechanical methods for determining roughness are widely used, for example, probe electromechanical devices like profilometers are used, and if necessary, the character of the irregularity along the length is recorded with profilographs. When choosing a construction material to use, in particular, from nanomaterials, it is necessary to take into account their toxicity, since it has to do with the pipelines of transport for both sewage and natural waters [7].

Investigations of the structure of the fluid near hydrophobic surfaces and, in particular, in the wall zone [8] have special interest.

The analysis of research in the field of static and dynamic wettability of various surfaces of building materials used in trenchless laying and renovation of pipeline networks contributed to focus on finding an effective protective coating of pipes with the identification of the relationship between the degree of hydrophobicity of the coatings and their hydraulic parameters, in particular, with roughness $[9,10]$. In connection with this, a compact test bench (open tray) was created and patented in the Moscow State University of Civil Engineering in order to determine the degree of hydrophobicity of materials for manufacturing pipes and repairing pipelines [11]. As a mechanism for calculating geometric and hydraulic parameters functioning according to the corresponding algorithm, a computer program was developed and patented [12].

The geometric and hydraulic characteristics of the mini-stream formed as a result of the flow of a certain mass of liquid through the open tray at various values of its slope, created by a special mechanical jack and controlled by a system of communicating tubes (Fig. 1) were studied at the stand. 


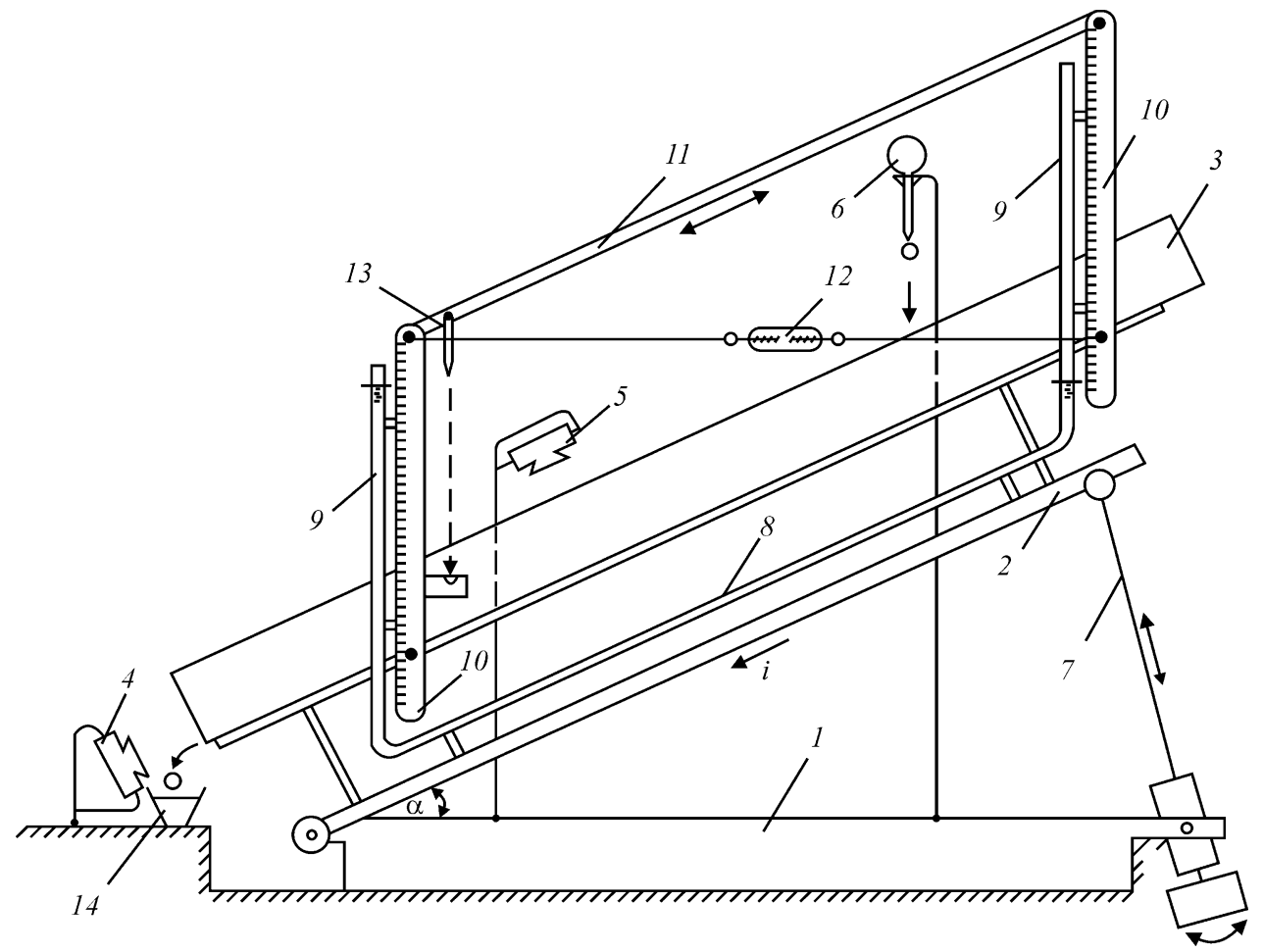

Fig. 1. Sketch diagram of the device of the test bench to determine the degree of hydrophobicity of materials

1- metal support frame; 2-ramp with a variable gradient; 3- open trench located on the trestle (tray) of the investigated pipe (about $1 \mathrm{~m}$ long); 4- the camera rigidly connected to the frame for frontal shooting; 5- the camera rigidly connected to the frame for co-axial shooting (fixing the length of the moving critical mass); 6 - a dispenser of liquid in the form of a pipette, 7 - a rod of a mechanical jack lifting an overpass; 8- transparent polyethylene line of the system of communicating vessels; 9 - transparent polyethylene tubes of the system of communicating vessels; 10- movable measuring rulers for fixing the water level in two tubes; 11- control bar for the position of the tubes and measuring rulers when the gradient changes; 12- screw cable ties; 13 - laser plummet; 14 - collection of drops (liquid flow); i - slope of the surface; $\alpha$ - the angle of the tray

During the experiments on various types of protective coatings used in trenchless construction and repair of pipelines, the parameters of the mini-stream were recorded using photo and film techniques. When conducting the experiments, a multi-frame serial shooting was carried out with the Sony SLR 550 (DT 1.8 / 50 SAM lens in conjunction with the Kenko Extension Tube system) and the Sony HDR-CX250 digital video camera.

The method of operation in the mentioned above installation is to compare the behavior of a mini-stream of a liquid (drinking water) on different working planes with different slopes of the sliding surface and to fix with measuring rulers and a stopwatch both the way and the rates of flow of the mini-stream through the tray. On the basis of the results of the experiment, visualized multimedia results are obtained in the form of digital photographs of the frontal and coaxial surfaces of the mini-stream. For the subsequent processing and interpretation of the data, the relationship between the volume and geometric parameters of 
the flow with the structure of a given surface is estimated, and, as a consequence, this relationship is extended to the parameters of the wetting regime in the form of a corner angle, referring the coating to hydrophilic or hydrophobic type, respectively [13].

Fig. 2 demonstrates an axonometric diagram of a mini-stream formed as a result of the flow of a critical volume of liquid on an inclined surface in the form of a tray. The flow is formed as a result of dosing a certain number of drops from the pipette onto the tray, which, when collected under the appropriate conditions (slope of the tray, the nature of the slip surface, etc.), form a critical mass starting to move along the inclined surface of the tray.

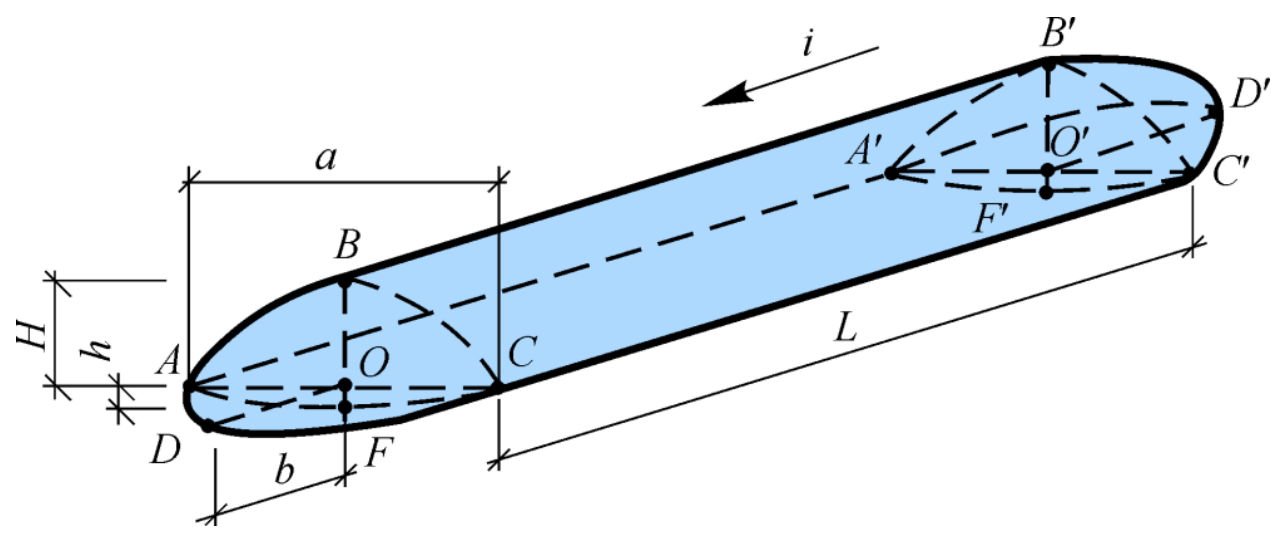

Fig. 2. Axonometric scheme of a mini-stream with geometric dimensions

$a, L$-respectively, are the average values of the width and length of the compact part of the ministream; $H, h$ - - respectively the height of the top and bottom lenses; $b$ - the height of the half of the ball segment of the head part of the mini-stream; AFC, $\mathrm{AA}^{\prime} \mathrm{C}^{\prime} \mathrm{C}$ - respectively wetted perimeter and wetted surface of the compact part of the mini-stream

Considering the cumbersome calculation of all intermediate geometric and hydraulic parameters, including the degree of hydrophobicity, an automated program is used, which dialog box is shown in Fig. 3.

According to the calculation algorithm, the parameters of fixation and subsequent automated processing are the following:

-The average length of the compact part of the mini-stream $L, m m$;

-The average width of the mini-stream $a, \mathrm{~mm}$;

- Top lens height (distance from the horizontal surface to the top of the lens) $\mathrm{H}, \mathrm{mm}$;

- Bottom lens height (distance from the tray to the horizontal surface) $h, \mathrm{~mm}$

- Number of drops forming a critical mass (mini-stream) $N$, pcs.

-Height of the head ball segment of the mini-stream $H_{g}, \mathrm{~mm}$

-The height of the tail ball segment of the mini-stream $H_{k}, \mathrm{~mm}$

- The path traversed by the head of the mini-stream $U, \mathrm{~mm}$

- Travel time $T, s$

The most significant practical effect obtained from the calculation results is the determination of a number of parameters, in particular the relative degree of hydrophobicity of the protective coating, the coefficients of Manning's and Chezy's relative roughness for the type of protective coating in question [14]. The presence of an automated program for calculating the above-described coefficients substantially accelerates and increases the accuracy of obtaining the required quantities. 


Calculation Of Protective Coatings
Calculation of protective coatings with an hydrophobicity degree
identification of pipe walls and protective coatings
Coating material: Polymer hose by Per Aarsleff (DEN)
The diameter of the tray d, mm:
Tray slope i:
Critical mass of water (number of drops) N. pieces:
The average length of the compact part of the mini-stream L, mm:
The mini-stream average width a, mm:
Top lens height $\mathrm{H}$, mm:
Bottom lens height $\mathrm{m}$, mm:
Height of the head ball segment of the mini-stream Hg. mm:
Height of the tail ball segment of the mini-stream Hk, mm:
The path traversed by the head of the mini-stream $\mathrm{U}$. mm:
Travel time $\mathrm{T}$, s:
$\begin{aligned} & \text { Microsoft Word } \\ & \text { Calculate and export to }\end{aligned}$

Fig. 3. The working interface of the automated program for the calculation of protective coatings with an hydrophobicity degree identification of pipe walls and protective coatings

Below there is a shortened final calculation of the geometric, hydraulic and hydrophobic characteristics of protective coating material made by Per Aarsleff (Denmark) as a result of the automated complex work. This type of protective coating is chosen as a model, which has found wide application in the field of renovation of water supply and drainage pipelines in many countries of the world [15].

Calculated data of output information after input according to Fig. 3:

1. The total volume of the mini-flow $W_{l}, \mathrm{ml}$ :

2. The mini-stream total volume $W, \mathrm{~mm}^{3}$ :

$$
W_{l}=0,0568 N+0,4943=1.232
$$

3. Top lens area $S_{v}, \mathrm{~mm}^{2}$ :

$$
W=10^{3} W_{l}=1232.700
$$

$$
S_{v}=2 a H / 3=23.253
$$


4. Bottom lens area $S_{n}, \mathrm{~mm}^{2}$ :

5. Small chord length $\mathrm{m}, \mathrm{mm}$ :

$$
S_{n}=2 a h / 3=5.086
$$

$$
m=\left[(a / 2)^{2}+h^{2}\right]^{1 / 2}=5.494
$$

6. Wetted perimeter $\mathrm{p}, \mathrm{mm}$ :

$$
p=2 m+(2 m-a) / 3=11.019
$$

7. Total area of the top and bottom lenses $S_{s}, \mathrm{~mm}^{2}$ :

$$
S_{s}=S_{n}+S_{v}=28.340
$$

8. Theoretical volume of the compact part of the mini-stream $W_{t}, \mathrm{~mm}^{3}$ :

$$
W_{t}=S_{s} L=510.120
$$

9. The wetted surface of the compact part of the mini-stream $S, \mathrm{~mm}^{2}$ :

10. Relative index of hydrophobicity $K_{o}$ :

$$
S=p L=198.349
$$

11. Edge angle (angle of wetting) $\alpha$, deg.:

$$
K_{o}=S_{v} /(S i)=1.0658
$$

$$
\alpha=49.6
$$

12. The estimated volume of the mini-stream $W_{r}, \mathrm{~mm}^{3}$ :

$$
W_{r}=W-(1 / 12)\left[H_{g}\left(H_{g}^{2}+0.75 a^{2}\right)+H_{k}\left(H_{k}^{2}+0.75 a^{2}\right)\right]=510.120
$$

13. Estimated length of the mini-stream compact part $L_{r}$, MM:

$$
L_{r}=W_{r} / S_{s}=18.051
$$

14. The approximation between the projected length $\mathrm{L}_{\mathrm{r}}$ and the average length $\mathrm{L}$ of the mini-stream $P_{L}, \%$ :

15. Tray filling $(h / d)$ :

$$
P_{L}=\left|100-L \cdot 100 / L_{r}\right|=0.287
$$

$$
(h / d)=h / d=0.00538
$$

16. Hydraulic radius $\mathrm{R}_{1}$ (through filling), $\mathrm{mm}$ :

$$
R_{l}=62.2(\mathrm{~h} / \mathrm{d})^{0,8833}=0.616
$$

17. Hydraulic radius $\mathrm{R}_{2}$ (through the wetted perimeter and live section), $\mathrm{mm}$ :

$$
R_{2}=S_{n} / p=0.461
$$

18. The absolute approximation value $P_{R}$ between $R_{l}$ and $R_{2}, \%$ :

$$
P_{R}=\left|100-R_{1} \cdot 100 / R_{2}\right|=33.488
$$

19. The adjusted exponent " $\mathrm{x}$ " in the formula to determine the hydraulic radius:

$$
R_{2}=62.2(h / d)^{X} ; \quad x=0.938
$$

20. The general corrected formula for determining the hydraulic radius $\mathrm{R}$ and its value, $\mathrm{mm}$ :

$$
R=62.2(h / d)^{X}=62.2(h / d)^{0.93859}=0.461
$$

21. Average mini-stream speed $\mathrm{V}, \mathrm{mm} / \mathrm{s}$ :

$$
V=U / T=16.40
$$

22. The Chezie's Coefficient $C$ :

$$
C=V /(R \cdot i)^{1 / 2}=72.779
$$

23. Manning's relative roughness coefficient $n$ :

24. Hydraulic friction coefficient $\lambda$ :

$$
n=(1 / C) R^{1 / 6}=0.0121
$$

$$
\lambda=8 g / C^{2}=0.0148
$$

The results of the automated calculation are output directly in Microsoft Word, which must be preinstalled on the user's computer (the version does not matter). The technology developed by Microsoft OLE (Object Linking and Embedding) is applied [16].

Making a conclusion of conducted researches and obtained results it is possible to note that thanks to the use of the developed technique, including a mathematical model 
describing the interaction of the structure of the investigated surfaces with the fluid, the algorithm and the automated program for calculating geometric, hydraulic and hydrophobic parameters, the researcher can get an overall picture of such indicators for any type of potential protective coating of pipelines subjected to trenchless repair or reconstruction.

\section{Conclusions}

1. On the basis of experimental studies, an automated calculation of the hydraulic parameters of one of the types of internal protective coatings used in technologies of trenchless construction and renovation of pressure and non-pressure pipeline systems was carried out.

2. According to an automated calculation, the Manning's roughness coefficient " $n$ " for the protective coating made by Per Aarsleff enterprise was equal to $\mathrm{n}=0.0121$, the hydraulic friction coefficient $\lambda=0.0148$, and the relative coefficient of hydrophobicity $\mathrm{Ko}=1.0658$. This coating should be attributed to the type of hydrophilic (the contact angle at the front section of the mini-stream was equal to 49.6 degrees).

3. The relatively low value of the degree of hydrophobicity (practically the lower limit in the range 1.06-2.0) of the studied material can indicate that, despite its wide use in practice of trenchless construction and reconstruction technologies, which should provide high strength characteristics of pipelines and guarantee the resource-saving effect, the material is surpassed by other types of protective coatings, which are hydrophobic type and provide a greater energy saving effect.

4. The proposed algorithm and automated program for calculating the hydraulic characteristics of protective coatings enable to make an operative evaluation of any types of materials that can be used in construction technologies.

\section{References}

1. A Kuliczkowski, Rury kanalizacyjne. Wydawnictwo Politechniki Swietokrzyskiej, p. 507 p., (2004)

2. S.V. Khramenkov, O.G. Primin, V.A. Orlov, Rekonstruktsiya truboprovodnyh system, ASV., 214 p., (2008)

3. L. Zhang, P. De Schryver, B. De Gusseme Chemical and biological technologies for hydrogen sulphide emission control in sewer systems, Water Research. 42. p. 1-12, (2008)

4. P. Roach, N.J. Shikcliffe, V.I. Newton, Progress in superhydrophobic surface development, The Roual Society of Chemistry, 4, 224-240 (2008)

5. T. Min, J. Kim., Effect of hydrophobic surface on stability and transition. Physics of fluids, 17, 106-108 (2005)

6. V.I. Margolin, V.A. Zhabriaev, G.N. Lukyanov, V.A. Tupik, Vvedenie v nanotekhnlogiyu, Lan', 457 p. (2012)

7. A.V. Kilesnitchenko, M.A. Timofeev, M.V. Protopopova, Toksichnost' nanomaterialov, Rossiyskie nanotekhnologii, 3, 3-4, 54-61 (2008) 
8. C. Choi, U.Ulmanella, J. Kim and C. Kim, Effect slip and friction reduction in nanograted superhydrophobic microchannels, Physics of fluids, 18, 087105 (2006)

9. S. Grossmann, D. Lohse, Curvature effects on the velocity profile in turbulent pipe flow, Eur. Phys. J. E., 40, 16 (2017)

10. C. Dorrer, J. Ruhe, Langmuir J., Condensation and Wetting Transitions on Microstructured Ultrahydrophobic Surfaces, 23, 3820-3824 (2007)

11. V.A. Orlov, I.S. Dezhina, E.V. Orlov, I.A. Averkeev, Ispytatelniy stend po opredeleniyu stepeni hydrofobnosti materialov diya izgotovleniyq trub $i$ remonta truboprovodov, Patent na poleznuyu model RF № 157695 (12015)

12. V.A. Orlov, S.P. Zotkin, I.S. Dezhina, A.A. Pelipenko, Programma rascheta stepeni hydrofobnosti $i$ hydravlitcheskih parametrov trub i zaschitnyh pokrytiy, Svidetelstvo o gosudarstvennoy registracii programmy dlya EVM № 2017612281 (2017)

13. L.B. Boynovich, A.M. Emelyanenko, Hydrofobnye materialy i pokrytia: principy sozdania, svoystva i primenenie, Uspekhi khimii Journal, № 77 (7), 621-638 (2008)

14. M.Rameil, Handbook of pipe bursting practice. Vulkan verlag, 351 p. (2007)

15. A. Kuliczkowski, E. Kuliczkowska, A. Zwierzchowska Technologie beswykopowe w inzeynierii srodowiska, Wydawnictwo Seidel-Przywecki Sp. 735 p. (2010)

16. S.P. Zotkin, B.S. Sadovskiy, I.A. Zotkina, K voprosu o sopryazhenii prikladnyh programm s Microsoft Word. Voprosy prikladnoy matematiki $i$ vychislitelnoy mekhaniki, Sbornik nauchnyh trudov MGSU, 17, p. 260-263 (2014) 